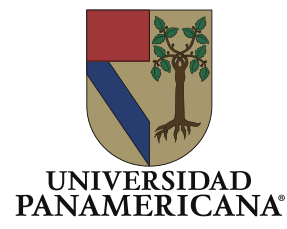

Available online at www.sciencedirect.com

ScienceDirect

BIOETHICS UPdate 6 (2020) 63-66
BIOETHICS UPdate

www.elsevier.es/bioethicsupdate

Editorial

\title{
The Coronavirus pandemic and the principle of common good
}

\author{
La pandemia del Coronavirus y el principio del bien común \\ Evandro Agazzi \\ Interdisciplinary Center for Bioethics, Panamerican University of Mexico, Mexico City, Mexico
}

The explosion of the Coronavirus pandemic has often produced opposite judgments and behaviors, depending on the authorities and the places where they were taken. This confusion was to some extent inevitable since it was a new and unknown virus, the aggressiveness and rapid spread of which were initially ignored. It is natural that they presented themselves in many urgent situations, in which patients with this new disease had to receive urgent therapies for which the healthcare facilities were unprepared. Hence the need to have guidelines and protocols available, especially to establish assistance priorities in the event of insufficient therapeutic tools available in practice. It was inevitable that ethical and, more specifically, bioethical issues would also emerge to guide urgent and often dramatic choices in such emergencies. We want to briefly mention some fundamental aspects of this problem.

Let us start by highlighting an attitude often present in the declarations of many personalities who showed up as "experts" especially in the mass media. They widely qualified as "heroes" all the medical and paramedical people engaged in the fight against Coronavirus. This eulogy - rightly addressed to many persons who assisted with full commitment and personal sacrifice these sick people - gradually became a kind of rhetorical tribute that ended up hiding several real aspects of the situation. In

E-mail address: evandro.agazzi@ gmail.com 
fact, it is not ethically correct to require that you have to be a hero in order to fulfill your duty: this conception would take away from the fulfillment of duty the aspect of a normal moral obligation, transforming it into an exceptional and not morally binding choice. In other words, the drama of the situation must highlight the real problem which is, at the same time, ethical and political, namely that of providing all healthcare workers with individual and environmental protections that allow them to perform their work without putting at risk their health and even their life. From this point of view, the protests and strikes of health personnel who refused to do their work because they had not been provided with the necessary protections by the competent institutions (or even had not managed to obtain them personally on the market), must be considered as a legitimate defence of personal rights to safety. The defence of such rights, however, is not a moral obligation of the single individuals concerned but is an ethical-political obligation of those who are committed to promote the common good and have the institutional and legal means for doing this at a public level.

Another problem, however, arises here: the various ethical and bioethical criteria that have been proposed and discussed by many parts are clearly set up from an individualistic perspective and, moreover, follow the lines of a utilitarian ethics quite explicitly. In fact, among the various ethical principles called into question, there is no reference to the common good, probably also due to the fact that it is not a concept that can be easily analyzed and translated into scores. However, some elementary examples can highlight its importance. Consider the case of the choice that must be made between offering the only respirator available at the moment to a young man of 20 or to a 70 -year-old researcher working in a biomedical laboratory. On the basis of the criteria defended today, preference is in favor of the former and, from a certain point of view, this choice is based on objective criteria such as the highest life expectancy and recovery possibility. However, the death of a researcher who is perhaps participating in the development of a therapy or of a vaccine entails, from the point of view of the common good of the community, a much greater damage than that of the loss of a young man in his early twenties. Life whose future developments are all unpredictable. This merciless speech also applies to the priority, practically accepted by all, to offer protection to medical and paramedical staff directly involved in anti-Coronavirus therapies. Everyone seems emotionally ready to subscribe to this choice, considering the "heroic" work of these people. There is, however, also a more objective reason, namely the fact that the failure of the work of this healthcare professionals (due to illness or death) removes irreplaceable capacities from the fight against Coronavirus which, from the point of view of the common good, deserves a very special consideration. At this point the analysis could become even more 
pitiless, recognizing that, while from the point of view of dignity and human rights the life of a doctor and that of a stretcher is on the same level, it is obvious that from an objective point of view, that is, from that of the common good, preserving the doctor is more important than protecting a stretcher bearer.

Other examples could be given and we limit ourselves to only one: the one in which it is a question of whether to guarantee the survival of the young 20-year-old or that of the 70-year-old pensioner, when the latter, unlike the former, is in practice the only source of income from which a family of five draws, which would remain without means of subsistence after his death. In this case, the criteria in circulation today strictly exclude that this type of condition be taken into consideration, yet from the point of view of the common good it is certainly not negligible.

There is no doubt that the foregoing considerations respect the sacrosanct principle that respect for a person is tied exclusively to what she is, and not to what she does. However, the principle of the common good must also bear in mind this second aspect and the difficulty lies precisely in making these two aspects compatible. Here the limits of a utilitarian perspective emerge. According to this, in fact, the moral goodness of an action is estimated on the basis of the fact that it produces the greatest possible degree of goods for the greatest possible number of individuals. These goods are actually of very different types and can range from health, wealth, success, job stability, pleasantness of material living conditions and so on. The strangeness of this criterion of morality consists in the fact that the moral goodness of an action is equated with the sum of many non-moral goods. Even the condition that this sum should benefit the maximum possible number of individuals - which seems to express the principle of the common good - is actually frustrated because, from the point of view of utilitarian ethics (which is consciously an individualistic ethics), the evaluation and weighting of the goods to be maximized depend on the personal preferences of the individuals and therefore is actually more a chimera than a usable criterion. The effort to analyze some of these "goods" objectively is quite different, and demanding, and should succeed in bringing at least a certain number of them into that patrimony of goods to whose enjoyment every human person has a moral right.

Imagining this difficulty resolved, it is immediately clear that the dramatic choices posed by the emergency situations mentioned above cannot be resolved without taking into account this complex set of values and precisely in this effort to optimize lies the difficulty of the moral judgment (which is very different from a simple calculation), that presides over the choice. Precisely for this reason it does not 
seem reasonable to imagine entrusting the choice to an administrative body, such as a "selection committee", simply charged with checking the correct attribution of scores. It is useful that these scores are somehow elaborated, but the final judgment must result from an integration carried out by the treating team which can turn, if it deems it necessary, to those ethics committees that should institutionally exist in hospitals, without need to delegate the competence to other bodies of an essentially bureaucratic and administrative nature. 